Original Research Paper

\title{
Risk Instrument for Screening in the Community (RISC): Cross Cultural Perspectives
}

\author{
${ }^{1}$ Patricia Leahy-Warren, ${ }^{1,2}$ Elizabeth Weathers, ${ }^{3}$ Marina Lupari, ${ }^{3}$ Sadie Campbell, \\ ${ }^{4}$ Roger Clarnette, ${ }^{5}$ Francesc Orfila, ${ }^{2}$ Christine Fitzgerald, ${ }^{6}$ Constança Paúl, ${ }^{2}$ Eileen O'Herlihy, \\ ${ }^{1,2}$ Nicola Cornally, ${ }^{2,7}$ Rónán O'Caoimh, ${ }^{1}$ Mary Rose Day, ${ }^{1}$ Helen Mulcahy and ${ }^{2}$ William Molloy \\ ${ }^{l}$ School of Nursing and Midwifery, University College Cork, Ireland \\ ${ }^{2}$ Centre for Gerontology and Rehabilitation, University College Cork, Ireland \\ ${ }^{3}$ Northern Health and Social Care Trust, Northern Ireland \\ ${ }^{4}$ Faculty of Medicine, Dentistry and Health Sciences, University of Western Australia, Australia \\ ${ }^{5}$ IDIAP Jordi Gol, Barcelona, Spain \\ ${ }^{6}$ ICBAS, University of Porto, Portugal \\ ${ }^{7}$ Health Research Board, Clinical Research Facility Galway, National University of Ireland, Galway, Ireland
}

Article history

Received: 29-01-2015

Revised: 11-08-2015

Accepted: 19-08-2015

Corresponding Author: Patricia Leahy-Warren School of Nursing and Midwifery, University College Cork, Ireland

Email: Patricia.Leahy@ucc.ie

\begin{abstract}
Older people are at an increased risk of developing multiple comorbidities causing subsequent cognitive, functional decline and frailty and increasing the risk of adverse healthcare outcomes. Public Health Nurses (PHNs), geriatricians and researchers developed the Risk Instrument for Screening in the Community (RISC) to record the presence of problems, severity (mild, moderate, severe) of concerns and caregiver networks' ability to manage the patient across three domains: mental state, activities of daily living and medical state. The aim of this study was to explore the experiences of healthcare professionals who had used the RISC with community-dwelling older adults, between countries. Five focus groups $(n=28)$ were conducted in five countries (Australia, Northern Ireland, Portugal, Republic of Ireland and Spain). Four main themes were identified from participants' experiences using the RISC: 'Tool Usability'; 'Benefits for Patients and Carers'; 'Operational Challenges'; and 'Recommendations for Improvement'. Some cultural differences were found between participants with regards to terminologies such as 'caregiver network' and 'insight' and expectation in relation to caring. Disciplinary differences were identified related to different conceptualisations of health. The RISC offers an innovative approach to identify and score risk in community dwelling older adults. However, further research is necessary to reflect cultural and disciplinary norms.
\end{abstract}

Keywords: Older Adults, Frailty, Risk Assessment, Primary Care

\section{Introduction}

Worldwide, populations are ageing and this will mean that growing numbers of people will be at risk of frailty. A systematic review found that the prevalence of frailty varies between countries, ranging from $4.0 \%$ to $59.1 \%$ (Collard et al., 2012). The diversity in this range can be in older adults attributed to differing definitions, as well as diversity within age and gender study cohorts. Frailty is associated with adverse healthcare outcomes (AO), including institutionalisation, hospitalisation and death. These AO can lead to significantly higher expenditures in health and social care (Slaets, 2006) and have become a priority for Health Care Professionals (HCP) and policy makers. Population wide screening and early identification of older adults, at risk of frailty and adverse outcomes, will enable triage and targeted priorities of social and health services and supports by HCPs in an effort to prevent or postpone these adverse outcomes. Internationally, nurses in the community use a variety of methods and assessments to identify frail older people in an attempt to identify the level of risk to prioritise anticipatory care (Ballard et al., 2013; Stijnen et al., 2014; Yoshioka-Maeda et al., 2006). O'Caoimh et al. (2015) developed and validated a short screening tool, The Risk Instrument for Screening in the
Science

Publications 
Community (RISC, Appendix 1), with public health nurses, for use in community dwelling older adults to screen for risk of frailty and AO. The RISC records the presence of concerns, the severity of concerns (mild, moderate and severe) and each caregivers' networks ability to manage these concerns in three domains: mental state, activities of daily living and medical state (O'Caoimh et al., 2015; O'Caoimh et al., 2012a; O'Caoimh et al., 2012b; O'Caoimh et al., 2013). There are benefits to seeking early international perspectives in instrument development (Poortinga, 2003), especially if there are plans to scale up these screening projects and use them in different countries, with variable cultures and health systems. The use of qualitative methods to ensure content validity and ease of completion of an instrument is recommended (Smith et al., 2011; Drennan, 2003). To capture the cross cultural understanding of the concept of frailty and its risks, the research team decided to explore the use of the RISC in five countries, to identify HCPs' experiences of its use, in clinical practice, at this early stage of development. The aim of this study was to explore the experiences of HCPs' in different cultures and healthcare delivery systems, who had used the RISC.

\section{Materials and Methods}

\section{Design}

A qualitative, descriptive design was used to provide a straight description of the experiences of those who had used the RISC. This design focuses on descriptive validity, to provide a comprehensive account of phenomena that both the researchers and participants would agree is accurate (Sandelowski, 2000).

\section{Settings and Participants}

A variety of health care settings were used to capture the diversity within which HCPs work and care for their older clients. Participants were recruited using convenience sampling from five clinical areas, in five different countries (Australia, Ireland, Northern Ireland, Portugal and Spain) who were piloting the RISC in older clients. HCPs were recruited if they were trained to use the RISC, had used the RISC with their clients, were proficient at reading and speaking English and were willing to participate. Prior to data collection, ethical approval was sought and granted by each participating local Clinical Research Ethics Committees.

\section{Ethical Considerations}

Ethical approval was sought and granted from each of the appropriate and relevant ethical committees.

\section{The RISC Instrument}

The Risk Instrument for Screening in the Community (RISC) is a short (2-5 min) instrument developed to identify those at greatest risk of three adverse outcomes, institutionalization, hospitalization and death (O'Caoimh et al., 2015; O'Caoimh et al., 2012a; O'Caoimh et al., 2012b; O'Caoimh et al., 2013). The instrument includes demographic data and records the presence (yes or no responses) and magnitude (mild, moderate, severe) of concern across three domains: mental state, ADLs and medical state. Based upon severity of concern and the caregiver networks' ability to manage the patient, an overall global risk score is then assigned to three adverse outcomes: institutionalization, hospitalization and death at one year from the date of assessment. In terms of assessment, five levels of risk from one (minimal and rare) to five (extreme and certain) are scored. The RISC instrument has excellent inter-rater reliability (Fleiss' Kappa $\mathrm{K}=0.86-1.0$ ) and internal consistency (Cronbachs` alpha coefficient, $\alpha=0.94$ ) and takes 2-5 min to complete (O'Caoimh et al., 2015; O'Caoimh et al., 2012a; O'Caoimh et al., 2012b; O'Caoimh et al., 2013).

\section{Data Collection and Analysis}

Data were collected using focus groups, which were audio recorded and analysed using thematic content analysis. Focus group interviews were transcribed in-full and data analysis was performed with the research team. To ensure rigour, each focus group discussion was framed around the semi-structured, open-ended questions, designed to ensure consistency across research sites. Significant statements and phrases from the transcripts were coded and clustered into categories and then collapsed into themes that were agreed by team members. Rigour was strengthened by the systematic collection, analysis and interpretation of the data (Graneheim and Lundman, 2004).

\section{Results}

The characteristics of the focus group participants are presented in Table 1. A total of 28 HCPs from five countries were involved. The majority of participants were female nurses. There were differences between countries in terms of the different disciplines involved in care of the older adult in the community setting.

Four main themes were identified from participant experiences of using the RISC. These were:

- Tool Usability

- Advantages of the RISC

- Operational Challenges

- Recommendations for Improvement

A number of subthemes were identified under each of these overarching themes (see Table 2). There were many similarities in experiences of participants across each of the five countries. However, some cultural differences were noted and these are included under the theme Operational Challenges. 
Table 1. Characteristics of study participants

\begin{tabular}{|c|c|c|c|c|}
\hline Country & $\begin{array}{l}\text { Setting where RISC } \\
\text { was used }\end{array}$ & Sample size & Discipline/role & $\begin{array}{l}\text { Experience working with } \\
\text { older adults (mean years) }\end{array}$ \\
\hline Northern Ireland & $\begin{array}{l}\text { Older people's homes } \\
\text { and residential care settings }\end{array}$ & $\mathrm{n}=8$ & $\begin{array}{l}\text { Senior nurse } \\
\text { Practitioners }(\mathrm{n}=8)\end{array}$ & $27^{*}$ \\
\hline Spain & Primary and community & $\mathrm{n}=7$ & Registered nurses $(\mathrm{n}=7)$ & 12.70 \\
\hline Australia & $\begin{array}{l}\text { Aged care assessment } \\
\text { Teams intertiary hospitals }\end{array}$ & $\mathrm{n}=4$ & $\begin{array}{l}\text { Social workers }(n=2) \\
\text { Clinical nurse consultant } \\
(n=1) \text { Geriatrician }(n=1)\end{array}$ & 21.75 \\
\hline Portugal & $\begin{array}{l}\text { Community and } \\
\text { primary care }\end{array}$ & $\mathrm{n}=8$ & $\begin{array}{l}\text { Nurse }(n=4) \\
\text { General Practitioner }(n=2) \\
\text { Geriatrician }(n=2)\end{array}$ & 18.33 \\
\hline Rep of Ireland & $\begin{array}{l}\text { Community and } \\
\text { primary care }\end{array}$ & $\mathrm{n}=5$ & $\begin{array}{l}\text { Public health nurses }(\mathrm{n}=4) \\
\text { assistant directors of public } \\
\text { health nursing }(\mathrm{n}=1)\end{array}$ & 10.50 \\
\hline
\end{tabular}

Table 2. Themes and subthemes identified

\begin{tabular}{ll}
\hline Theme & Subtheme \\
\hline Tool usability & Scoring \\
& Easy to use \\
Advantages of the tool & Comprehensive and holistic \\
& Predictor of patient needs \\
& Empowerment of healthcare professionals \\
Operational challenges & Focus on caregiver network \\
& Communication \\
& Variation in scoring \\
Recommendations for improvement & Sensitive topics \\
& Patient history \\
& Cultural Issues \\
& Content, structure and format \\
\end{tabular}

\section{Tool Usability}

Participants in all of the five countries referred to the usability of the tool and particularly emphasised ease of scoring, ease of use and comprehensiveness.

\section{Scoring}

Participants in each of the countries alluded to the difficulty in providing an overall subjective score for risk of adverse outcomes. One participant in Spain referred to the problem of competing factors outside of those measured in the RISC:
"At certain ages, death seems to me especially difficult to score, with patients older than [90] and possibly in a stable situation but it would not surprise me that they died because they are that age..."

Similarly in Ireland, another participant discussed the problem of a subjective overall score:

"I suppose it was the predicting thing, it was a whole new thing to us."

Likewise participants in Portugal described the 'challenge' of making subjective decisions in relation to scoring and predicting adverse outcomes.
One Spanish participant considered the use of a 5-point scale better than a categorical yes/no response option:

"Compared to another tool we have to predict death 'yes/no', here the 5 categories to score risk look better."

\section{Easy to use}

All participants reported that the RISC was easy to use. Some participant quotations from each country include:

"I found it easy and logical..." (Spain);

"...easy to use, self-explanatory, could be utilised in a variety of settings" (Australia); "...straightforward form to use...covered everything and it was very efficient" (Ireland); "...the speed with which it can be completed but also capturing all the necessary information" (Northern Ireland);

"I enjoy using it" (Portugal).

\section{Comprehensive and Holistic}

Although participants reported that the tool was quick and easy to use, they also felt that it was comprehensive, covering a wide range of domains that provided a holistic view of the patient. One Irish participant reported that the RISC: 
“...incorporates all aspects of the patient, the physical, psychological, social and carer's needs in a very concise format."

Similarly, an Australian participant stated that the RISC:

“...enables thorough assessment and a focus on the caregiver network."

\section{Advantages of the RISC}

The reported advantages of the RISC were not specific to each country. These included the ability of the RISC to predict patient needs; empower HCPs; focus on the caregiver network; and enhance communication between HCPs, patients and families.

\section{Predictor of Patient Needs}

Many participants across countries referred to the usefulness of the RISC in predicting patient needs. Participants in Australia stated that the RISC allowed assessors to plan for the future care needs of the patient depending on their degree of risk. One Irish participant reported that the RISC should be a standard compulsory assessment:

"... if you did the RISC form so that at least the next person coming along [would have an overview of the patient's current status], [and if] it was done regularly, otherwise information goes with the public health nurse and is lost."

Another Irish participant alluded to the accuracy of the predictions:

$$
\text { “... what I predicted actually did happen." }
$$

\section{Empowerment of Healthcare Professionals}

The RISC enabled HCP so classify patients into low, medium and high risk. One Spanish participant stated:

"It is easy to use and all patients have a structured form, you can stratify the risk"

Another participant in Ireland said:

"It was very good in helping to categorise the elderly"

Consequently, HCPs were then able to prioritise care:

"... it quickly determines if someone is at risk of the adverse outcome. I can then prioritise who to refer to as soon as possible to try to maximise the benefits of the risks that I have identified" (Northern Ireland participant).

Similarly, a Spanish participant stated that the RISC:

"Helps you to organize your patients based on levels of risk."
With the RISC, HCPs were able to ensure that all those at high risk of adverse outcome, were promptly referred to the most appropriate service or intervention. An Australian participant discussed how the RISC enabled them to intervene appropriately, for example to arrange respite or an increase in care. Similarly, a Northern Ireland participant said:

"By using RISC, I can keep on my caseload those I can help the most and signpost the others to an appropriate pathway".

The RISC also empowered HCPs through enhanced inter-disciplinary communication, by providing objective evidence of those in greatest need:

"It's good when we have to go to other disciplines for services or signposting, we have evidence of those in greatest need" (Participant from Ireland).

Likewise, a participant from Northern Ireland stated:

"I work better with the GPS at identifying those most at risk".

\section{Focus on Caregiver Network}

Another benefit of the RISC was that it recognised the importance of caregivers and the caregiver network in predicting adverse outcomes. In Spain participants commented on this topic:

"The social network of the caregiver is very important..."

"If the caregiver fails, then it is when you have admissions, hospital, long term care..."

"The patient may be in good form, with no caregiver but no social network, they may get depressed and have an admission..."

\section{Communication}

Some participants described how using the RISC opened up communication pathways between HCPs, patients and families. Participants in Portugal reported that the RISC helped HCPs and families plan for the future, in terms of potential changes to health. Similarly, a participant in Ireland said:

"It was a great exercise for us as well in making contact with every family"

\section{Operational Challenges}

A number of operational challenges were identified by participants across all five countries. Some were specific to one or two countries. These challenges included variation in scoring, the sensitive nature of the items, the need to have a good understanding of the patient's history and specific cultural issues. 


\section{Variation in Scoring}

Portuguese participants emphasised the variation in scoring the RISC:

"...depending on the role of the health professional, scores varied. For example, with ADLs nurses scored higher than doctors and with medical issues, doctors scored higher than nurses",

Thus, clinical background factors appeared to impact on scoring. Also age might cause variation in scoring:

$$
\begin{aligned}
& \text { "...older doctors scoring higher and younger } \\
& \text { doctors scoring lower" (Portuguese } \\
& \text { participant). }
\end{aligned}
$$

\section{Sensitive Topics}

In Northern Ireland, participants described the difficulty in discussing sensitive issues such as mental health, with patients:

"Can be difficult to approach mental health issues on a first visit to someone who does not know you."

Some participants suggested that the order of the questions on the RISC should be altered to begin with easier questions e.g. ADL, leaving mental health questions until last:

"the ADLs should be domain 1, medical domain 2 and the mental health one 3rd" (Northern Ireland participant).

Another participant in Northern Ireland concurred:

"I feel it is more appropriate to commence any assessment with the person's physical condition"

The issue of discussing the findings of the RISC with patients was also raised. HCPs did not know how to communicate results with patients. A participant in Northern Ireland stated:

"How do we explain this to patients? What are the implications of telling them of the adverse outcome in one year?"

Another participant concurred:

"I wasn't sure what I would say to the patient if their risk of death was high" (Northern Ireland participant).

\section{Patient History}

Many participants discussed the need to be familiar with patients and to have some knowledge of their medical background:

\begin{abstract}
"I feel also if I am meeting the person for the first time I like to find out a bit of background history on medical conditions or previous hospital admissions before carrying out any assessment, therefore I would like a small area on the form to capture this information and somewhere to record clinical observations" (Northern Ireland participant).
\end{abstract}

Similarly, a participant in Ireland reported that it was easier to score patients who they knew for a long time or visited regularly:

\section{"You could predict the ones you knew very well, but for others it would be difficult."}

This problem was particularly relevant to public health nurses who moved from one geographical area to another, as is reflected in the following quotes:

"I don't know how easy it would be to do the predicting if you'd just come into an area" (Participant in Ireland);

"I had been in the area for a few years, but there was still a few people I didn't know" (Participant in Ireland).

\section{Cultural Issues}

A number of culturally specific issues were identified. Portuguese participants highlighted translation issues:

"Some terms don't translate very well or at all from the CARTS programme in Portuguese, such as 'insight', where no word in Portuguese exists to describe this..."

Portuguese participants also reported confusion over terms such as 'frailty' and 'risk' that aren't widely used or understood by HCPs there. Interestingly, also in Portugal, the role of the family was especially important in predicting risk of institutionalisation and in particular the role of females. Participants stated that daughters or daughters-in-law are considered responsible for caring for family members in their own home. There was stigma associated with nursing home placement in Portugal and it was only considered as a last resort.

\section{Recommendations for Improvement}

In all five countries some recommendations for improvement were made by participants. These pertained to content, structure and format and to the need for training and education on the use of RISC.

\section{Content, Structure and Format}

Participants in Ireland, Northern Ireland and Spain made several comments on the content, structure and format of the tool. With regard to content, some participants in Ireland identified the importance of 
environment which was said to have a 'massive impact' on the independence and lives of older people. The geographical location of patients impacted on the services they could access as reported by the following Irish participant:

"We are very near the main hospitals within the catchment of all acute services.. but if you are out in more rural areas maybe transportation would be an issue."

It was suggested that a new heading focusing on environment with examples such as access to services and transport, should be included:

"Environment I would think absolutely, I think it should be a tick box on the form" (Irish participant).

Similarly, Spanish HCPs identified the need for more prompts and examples under each dimension:

"Should be more concrete about what it is talking about, in each dimension, feel the need to specify more."

In relation to structure, participants in Northern Ireland identified the need for an extra column on the tool to allow HCPs to document a future review of the scores. Another Northern Ireland participant added:

\section{"A larger blank area at Step 2 stage to enable some free hand writing. This would mean all information captured could remain on the one form."}

In terms of format, one Northern Ireland participant commented:

"The writing is quite small making the page look busy."

\section{Training and Education}

The need for training and education on how to use the RISC and interpretation of the results was identified by many participants across the five countries. In Ireland, one participant stated:

"You'd certainly need some sort of an explanation around it."

Some participants in Portugal identified a lack of confidence in using the RISC. In Spain, one participant said:

"I thought we should need more information... and also knowing the patient's situation and carer better...".

Furthermore, some participants in Portugal and Ireland commented that the training cases not being entirely reflective of clinical practice:
"It was much easier on the real cases because you knew them".

\section{Discussion}

The aim of this study was to gain insight into the experiences of HCPs using the RISC tool across five countries. The five countries differ greatly in terms of culture and healthcare delivery systems. Four of the countries were European and the fifth site was in Australia. There were similar concerns and issues across all of the five countries and four main themes emerged from the data. These pertained to the usability of the tool, the advantages of the tool, challenges in operationalization and recommendations to improve the instrument. A number of sub-themes were also identified under each of the main themes. In relation to the usability of the tool, many participants identified problems with scoring, especially assigning an overall global risk score. Although prior research has provided evidence for the validity and reliability of the instrument (O'Caoimh et al., 2012a), it requires refinement and validation in other cultures and languages, particularly the establishment of cut off-points to identify low, medium and high risk in these populations. The research team are already investigating these issues further. In terms of instrument development, the goal is to ensure that an instrument is quick and easy to use, while also ensuring the content of the instrument captures all necessary domains (DeVellis, 2012). One of the strong points of the RISC is that, across all five countries, participants reported it as easy to use. Furthermore, only a short amount of time was required to complete it. Yet, the RISC was also reported as comprehensive and holistic.

The second theme that emerged highlighted the advantages of the RISC. Firstly, this referred to the ability of the RISC to predict patient needs. Prior research indicates that the majority of older people living in the community are independent and well (Kamiya et al., 2012). Yet, there are some older people living in the community who have may have complex physical/medical, or mental health needs and may not have a secure caregiver network. The benefits of targeting anticipatory care have been found to reduce adverse outcomes and hospitalisations (Hogg et al., 2009). Hence, there is a need for early intervention with this high-risk group of older people. The RISC has potential to be used by HCPs working in the community to screen patients for risk of hospitalisation, institutionalisation and death. In terms of the ability of the RISC in predicting outcomes, prior research shows that it has comparable accuracy (O'Caoimh et al., 2015) to the Clinical Frailty Scale, a well validated frailty measure (Rockwood et al., 2005).

Another advantage of the RISC identified by participants was the empowerment it gave them. Namely, the tool enabled them to stratify risk and 
identify those patients who were most in need. HCPs working in the community could then use this objective data to refer the patient to other healthcare professionals, or to enact a particular care pathway, based on the identified risk. This is an important advantage. Often, for older people in the community, it is the public health nurse who has most contact with them. Home visiting is an integral aspect of PHN practice and vulnerable older adults are visited more frequently by PHNs (McGee et al., 2008). PHNs are ideally placed to identify frail, housebound older adults who are vulnerable and at risk for hospitalization and morbidity (Ballard et al., 2013). However, it is difficult to ensure appropriate referral or intervention without objective data. The RISC addresses this gap. Aside from inter-disciplinary communication, the RISC also improved communication between healthcare professionals, patients and their families.

Another advantage of the RISC is that it places emphasis on the caregiver network and the importance of this network in predicting adverse outcomes of patients. According to O' Caoimh et al. (2014) there is a significantly increased risk of adverse outcomes where caregiver networks are regarded as dysfunctional, i.e., "under strain/not managing". This is particularly significant for institutionalisation risk where caregiver strain is known to increase risk of institutionalisation (McCann et al., 2011).

The third and fourth themes referred to operational challenges and recommendations for improving the RISC. Participants reported that scores varied depending on which discipline they came from and how old they were. Other operational challenges identified included the sensitive nature of some of the questions. Suggestions were made regarding the structure of the RISC. The research team have taken these suggestions into consideration and the research team will consider in further development of the instrument. Another challenge identified was communicating the results of the screening to patients, particularly if a person was identified as high risk of hospitalisation, institutionalisation, or death. This is something which could perhaps be probed further during the training provided to healthcare professionals.

The need for healthcare professionals to be familiar with the patients they were screening was another challenge identified. Staff turnover and movement of staff across geographical regions impacted on this. One recommendation made was to establish the RISC assessment as a compulsory assessment prior to handing over a caseload in order to provide a baseline risk score. Other issues pertained to the cultural translation of the RISC and training material. Participants in Portugal and Spain in particular identified a lack of confidence and a need for further training. Perhaps, this indicates the need to allocate extra time and resources for training in non-English speaking countries in comparison to English speaking countries. Also, it would be important to assess the learning of participants in non-
English speaking countries after training to ensure that their learning needs have been met. The final theme that emerged from the data outlined recommendations for improvement of the RISC. These specifically pertained to content, structure and format and the importance of training and education on the use of the RISC.

This study demonstrates the value of using qualitative methods e.g. quality enhancement groups, focus groups, in instrument development and adaptation of instruments. By default, the findings of this research helped establish the content validity of the instrument as well as any practical issues in terms of completion. Additionally, the findings highlight the advantages of the screening tool and further support the need for an instrument such as the RISC.

\section{Conclusion}

In conclusion, this study evaluated the experiences of healthcare professionals who used a short, global, subjective assessment to score for the risk of hospitalisation, institutionalisation, or death with older adults living in the community. Participants were from five countries. The findings suggest that the RISC tool has many advantages as a screening instrument for use in the community and is applicable cross-culturally. Future studies should focus on further psychometric testing of the RISC.

\section{Acknowledgements}

We would like to thank most sincerely the healthcare professionals who participated in our study for taking time to share their experiences of using the RISC instrument.

\section{Author Contributions}

PLW was the principal investigator. All authors contributed to study design. This study was conducted in five countries-all authors were responsible for data collection in their corresponding country. All authors contributed to the interpretation of findings and have approved the final manuscript.

Patricia Leahy-Warren: Contributed to study design, data collection, analyses, preparation, development and publication of this manuscript.

Elizabeth Weathers: Contributed to the preparation, development and publication of this manuscript.

Marina Lupari: Contributed to the preparation, development and publication of this manuscript.

Sadie Campbell: Contributed to study design, data collection, preparation, development and publication of this manuscript. 
Roger Clarnette: Contributed to study design, data collection, preparation, development and publication of this manuscript.

Francesc Orfila: Contributed to study design, data collection, preparation, development and publication of this manuscript.

Christine Fitzgerald: Contributed to data collection, preparation, development and publication of this manuscript.

Constança Paúl: Contributed to study design, data collection, preparation, development and publication of this manuscript.

Eileen O'Herlihy: Contributed to the preparation, development and publication of this manuscript.

Nicola Cornally: Contributed to the preparation, development and publication of this manuscript.

Rónán O'Caoimh: Contributed to study design, preparation, development and publication of this manuscript.

Mary Rose Day: Contributed to the preparation, development and publication of this manuscript.

Helen Mulcahy: Contributed to the preparation, development and publication of this manuscript.

William Molloy: Contributed to study design, data collection, analyses, preparation, development and publication of this manuscript.

\section{Ethics}

Authors should address any ethical issues that may arise after the publication of this manuscript.

\section{References}

Collard, R.M., H. Boter, R.A. Schoevers and R.C. Oude Voshaar, 2012. Prevalence of frailty in community-dwelling older persons: A systematic review. J. Am. Geriatrics Society, 60: 1487-1492. DOI: $10.1111 / \mathrm{j} .1532-5415.2012 .04054 . \mathrm{x}$

Slaets, J.P., 2006. Vulnerability in the elderly: Frailty. Med. Clin. North Am., 90: 593-601. PMID: 16843764

Ballard, J., M. Mooney and O. Dempsey, 2013. Prevalence of frailty-related risk factors in older adults seen by community nurses. J. Advanced Nursing, 69: 675-84. DOI: $10.1111 / \mathrm{j} .1365-2648.2012 .06054 . \mathrm{x}$

Stijnen, M.M., H.J. Vrijhoef, I.G. Duimel-Peeters and M.W. Jansen, 2014. Formulation of and adherence to a care plan for potentially frail community-dwelling older people by practice nurses in the Netherlands. J. Nursing Education Practice, 4: 34-43. DOI: $10.5430 /$ jnep.v4n8p34

Yoshioka-Maeda, K., A. Taguchi, S. Murashima, K. Asahara and Y. Anzai et al., 2006. Function and practice of public health nursing in Japan: A trial to develop the Japanese purpose-focused public health nursing model. J. Nursing Management, 14: 483-489. DOI: $10.1111 / \mathrm{j} .1365-2934.2006 .00688 . \mathrm{x}$
O’Caoimh, R., Y. Gao, A. Svendrovski, E. Healy and E. O'Connell et al., 2015. The Risk Instrument for Screening in the Community (RISC): A New Instrument for Predicting Risk of Adverse Outcomes in Community Dwelling Older Adults. BMC Geriatrics, DOI: 10.1186/s12877-015-0095-z

O'Caoimh, R., E. Healy, E. O Connell, Y. Gao and D.W. Molloy, 2012a. The Community Assessment of Risk Tool, (CART): Investigation of inter-rater reliability for a new instrument measuring risk of adverse outcomes in community dwelling older adults. Irish J. Med. Sci., 181: 227-227.

O'Caoimh, R., E. Healy, E. O Connell and D.W. Molloy, $2012 \mathrm{~b}$. Stratification of the risk of adverse outcomes for Irish, community dwelling, older adults: Use of a risk register. Irish J. Med. Sci., 181: 295-295.

O'Caoimh, R., Y. Gao, E. Healy, E. O'Connell and G. Gabrielle O'Keeffe et al., 2013. Screening for markers of frailty and percieved risk of adverse outcomes using the community assessment of risk tool. Irish J. Med. Sci., 182: 230-230.

Poortinga, Y., 2003. Cross-cultural Assessment. In: Encyclopedia of Psychological Assessment, Ballesteros, R.F., (Ed.), SAGE Publications, London, ISBN-10: 0761954945, pp: 1164.

Smith, M.L., E.T. Sosa, C.A. Tisone and E.L.J. Mckyer, 2011. Quality enhancement groups: A qualitative research method for survey instrument development. J. Health Behavior Public Health, 1: 15-22.

Drennan, J., 2003. Cognitive interviewing: Verbal data in the design and pretesting of questionnaires. J. Advanced Nursing, 42: 57-63. DOI: $10.1046 /$ j.1365-2648.2003.02579.x

Sandelowski, M., 2000. Combining qualitative and quantitative sampling, data collection and analysis techniques in mixed-method studies. Res. Nurs. Health, 23: 246-255. PMID: 10871540

Graneheim, U.H. and B. Lundman, 2004. Qualitative content analysis in nursing research: Concepts, procedures and measures to achieve trustworthiness. Nurse Educ. Today, 24: 105-112. PMID: 14769454

DeVellis, R.F., 2012. Scale Development: Theory and Applications. 1st Edn., SAGE, Thousand Oaks, ISBN-10: 1412980445, pp: 205.

Kamiya, Y., C. Murphy, G. Savva and V. Timonen, 2012. Profile of community-dwelling older people with disability and their caregivers in Ireland. TILDA, Trinity College Dublin.

Hogg, W., J. Lemelin, S. Dahrouge, C. Liddy and C.D. Armstrong et al., 2009. Randomized controlled trial of anticipatory and preventive multidisciplinary team care: For complex patients in a communitybased primary care setting. Can. Fam. Physician, 55: e76-e85. PMID: 20008582 
Rockwood, K., X. Song, C. MacKnight, H. Bergman and D.B. Hogan et al., 2005. A global clinical measure of fitness and frailty in elderly people. Can. Med. Association J., 173: 489-495. PMID: 16129869

McGee, H.M., A. O'Hanlon, M. Barker, A. Hickey and A. Montgomery et al., 2008. Vulnerable older people in the community: Relationship between the vulnerable elders survey and health service use. J. Am. Geriatr. Soc., 56: 8-15.

DOI: $10.1111 / \mathrm{j} .1532-5415.2007 .01540 . \mathrm{x}$
O’Caoimh, R., Y. Gao, A. Svendrovski, E. Healy and E. O'Connell et al., 2014. Screening for markers of frailty and perceived risk of adverse outcomes using the Risk Instrument for Screening in the Community (RISC). BMC Geriatrics, 14: 104-104. DOI: $10.1186 / 1471-2318-14-104$

McCann, M., M. Donnelly and D. O'Reilly, 2011. Living arrangements, relationship to people in the household and admission to care homes for older people. Age Ageing, 40: 358-363.

DOI: 10.1093/ageing/afr031
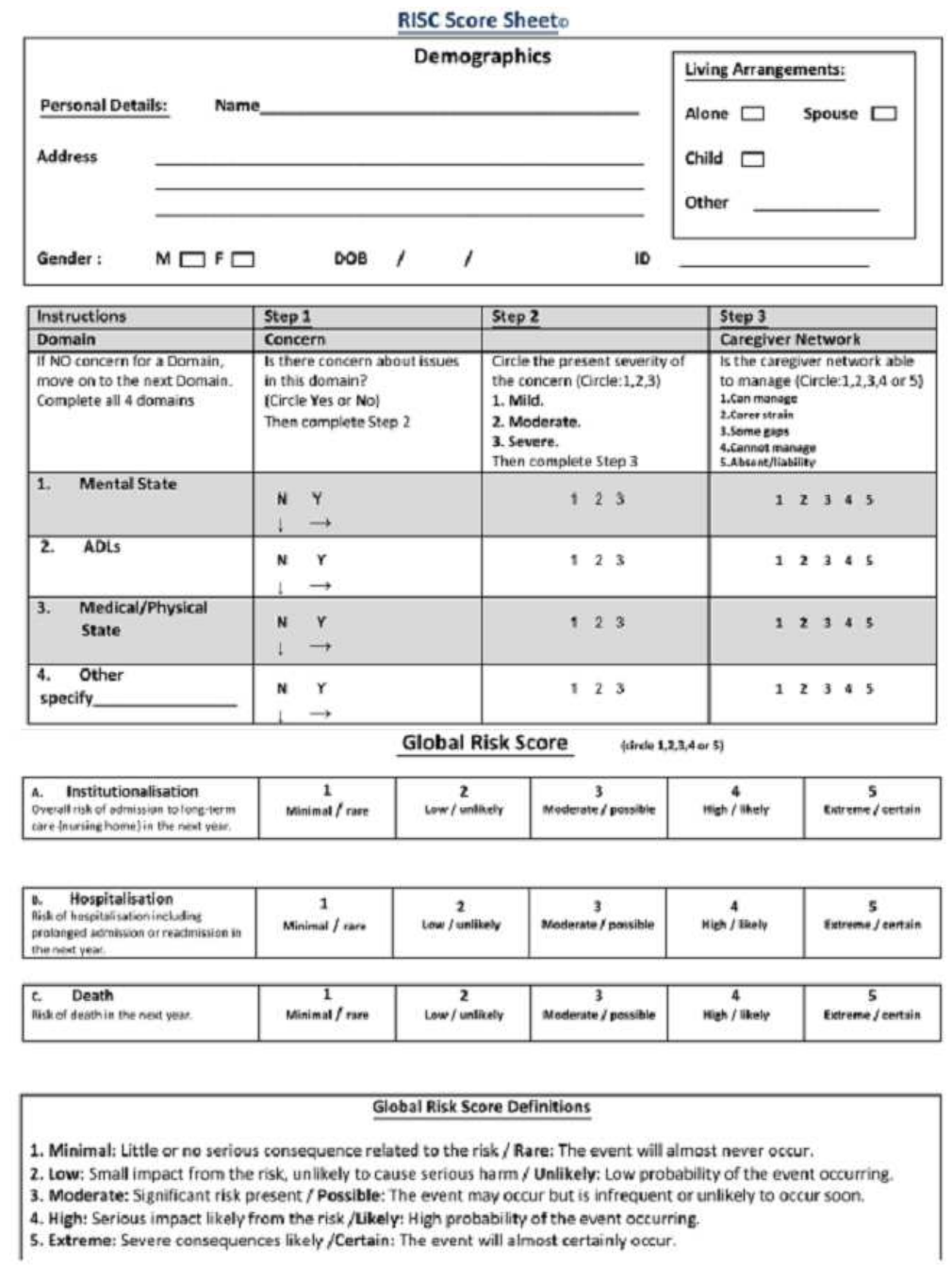

(7) OCaoimh\& Molloy 2013

Appendix 1. RISC Score SheetC available at http://www.biomedcentral.com/1471-2318/14/104/figure/F1 